\title{
Suppression of the inflammation and fibrosis in Asherman syndrome rat model by mesenchymal stem cells: histological and immunohistochemical studies
}

\author{
Nagla Mohamed Salama ${ }^{1}$, Somaia Saad Zaghlol', \\ Hala Hassan Mohamed ${ }^{1}$, Samaa Samir Kamar ${ }^{1}$ \\ ${ }^{1}$ Department of Histology and Cell Biology, Faculty of Medicine, Cairo University, Cairo, Egypt
}

\begin{abstract}
Introduction. Asherman syndrome (AS) is a symptomatic intrauterine adhesion caused by endometrial basal layer fibrosis as a result of either uterine cavity surgery or infection leading to many complications. There is a concern to repair the injured tissues by using bone marrow mesenchymal stem cells (BM-MSCs). We aimed in this study to develop an animal model of AS and evaluate the anti-inflammatory and anti-fibrotic effects of BM-MSCs in this model through histological, immunohistochemical, and morphometric studies.

Material and methods. Forty-two adult female adult albino rats were divided into (i) donor group composed of 2 rats used for isolation and propagation of BM-MSCs, and (ii) experimental groups: 40 rats equally divided into 4 groups: GpI (control), GpII (AS model), GpIII (BM-MSCs-treated AS rats), GpIV (untreated AS rats). Histological staining and immunohistochemical (IHC) detection of proliferating cell nuclear antigen (PCNA), vascular endothelial growth factor (VEGF), and nuclear factor-kappa beta (NF- $\kappa$ B) were performed. The results were evaluated by morphometric and statistical analysis.

Results. Significant endometrial thinning, fibrosis, and degeneration of the endometrial epithelium with a significant decrease in PCNA and VEGF immunoexpression and a significant increase in NF- $\kappa$ B immunoexpression were detected in GpII and GpIV groups. These changes were substantially reversed in BM-MSCs-treated animals. Conclusions: BM-MSCs treatment resulted in substantial improvement of intrauterine adhesion in the rat model of Asherman syndrome. (Folia Histochemica et Cytobiologica 2020, Vol. 58, No. 3, 208-218)
\end{abstract}

Key words: BM-MSCs; intrauterine adhesions; NF- $\kappa$ B; PCNA; VEGF; IHC

\section{Introduction}

Asherman syndrome (AS) is a symptomatic intrauterine adhesion (IUA) caused by endometrial basal layer fibrosis as a result of either uterine cavity surgery or infection. This can lead to partial or complete obstruction of the uterine cavity with abnormal menstruation such as amenorrhea, hypomenorrhea, and menorrhagia [1]. Besides, it could potentially

Correspondence address: Samaa Samir Kamar,

Department of Histology and Cell Biology, Faculty of Medicine

Cairo University, Cairo, Egypt

phone: +02 01008909069

e-mail: Dr_samaakamar@yahoo.com corrupt the blastocyst implantation and impede blood supply to the uterus and embryo, causing recurrent miscarriage or infertility [2]. The incidence of AS has been increasing over the last few decades because of the expanding frequency of cesarean sections and uterine medical procedures [3]. The main pathologic issue is the avascular fibrous connective tissue (CT) band with or without glandular tissue [4]. The current treatment strategy of AS includes dilatation and curettage, uterine balloon stent, and hormonal treatment to restore normal endometrium [5]. However, this strategy is characterized by a high failure rate due to the recurrence of the adhesion [6].

The use of bone marrow mesenchymal stem cells (BM-MSCs) opens a new era in repairing the tissue 
injury. BM-MSCs are adult stem cells that have the ability of self-renewal, transdifferentiation, and autotransplantation without stimulation of immune rejection [7]. Mesenchymal stem cells (MSCs) have provided a novel method in the treatment of fibrotic disease due to their ability to evade the immune detection and secretion of anti-inflammatory and anti-fibrotic mediators [8]. After autotransplantation, they can migrate and accumulate in the endometrial tissue. Thus, BM-MSCs treatment may be a promising therapy for AS [9].

The development of an animal model of AS is particularly essential for the study of AS phenotype, pathogenetic mechanisms, and the development of effective therapeutic strategies for the treatment of AS [2]. Trichloroacetic acid (TCA) is a topically applied chemical agent that is not absorbed systemically and denatures proteins, resulting in chemical cauterization. Uterine TCA installation is a chemical ablation technique that has been used previously in humans to treat dysfunctional menstrual bleeding [10]. It has been postulated that TCA could be used experimentally to develop a model of AS in rat [11].

The aim of this study was to evaluate the possible therapeutic anti-inflammatory and anti-fibrotic effects of BM-MSCs in experimentally induced AS model using histological, immunohistochemical and morphometric studies.

\section{Materials and methods}

Design of the experiment. This study included 42 adult female Wistar albino rats (aged about 12 weeks), with average body weight 200-220 g. Each group was kept in separate wire cages at room temperature (about $25^{\circ} \mathrm{C}$ ) in $12: 12 \mathrm{~h}$ light/dark cycle, fed ad libitum with free water access. All procedures of the study protocol were approved by the Institutional Animal Care and Use Committee of Cairo University (CU-IACUC) with an approval number (CUIII-F-28-18) and the principles of laboratory animal care. Animals were divided into donor group and experimental groups. The donor group included 2 rats used for stem cell isolation, culture, phenotyping, and labelling.

The rats of the experimental groups were cycle synchronized according to the vaginal smear examination. Daily morning vaginal smear was performed and analyzed to assess the estrous cycle. Rats with 4-5 days regular cycles were included in the experiment. 40 rats were included and equally divided into the following four groups $(\mathrm{n}=10 \mathrm{each})$.

GpI (control): The animals were equally subdivided into 2 subgroups $(\mathrm{n}=5$ each $)$ :

- subgroup Ia: normal rats, not subjected to any surgical procedure;

- subgroup Ib: rats underwent a sham operation in which the right (Rt) uterine horn was installed with $0.3 \mathrm{ml}$ saline.
GpII (AS model): the rats were anesthetized using an intraperitoneal injection of ketamine $(50 \mathrm{mg} / \mathrm{kg})$ and placed in a supine position. The inferior abdomen was shaved and disinfected, then a midline incision (about $3 \mathrm{~cm}$ ) was made. For the trichloroacetic acid (TCA) injection (Istanbul Ilac Sanayive Ticaret AS, Turkey), each uterine horn was identified and clamped with hemostatic forceps. Rt uterine horn was injected with a single dose of $0.3 \mathrm{~mL}$ of $90 \%$ TCA using an insulin needle. The peritoneum was irrigated with Ringer solution and the abdominal wall was closed. Post-operative analgesics and prophylactic antibiotics were supplied. Rats were sacrificed in the estrus phase of the $3^{\text {rd }}$ cycle (about 2 weeks) to assess the establishment of the model [10,11].

GpIII (AS rats BMSCs-treated): after 2 weeks of the modeling as in GpII, $1 \times 10^{6}$ PKH26 labeled BM-MSCs suspended in $1 \mathrm{ml}$ of phosphate-buffered saline (PBS) were injected into the tail vein of each rat. Then the animals were sacrificed in the estrus phase of the $3^{\text {rd }}$ cycle [12].

GpIV (untreated AS): after 2 weeks of modeling, each rat was injected via tail vein with $1 \mathrm{ml}$ PBS (solvent of BMMSCs). They were then sacrificed in the estrus phase of the $3^{\text {rd }}$ cycle.

Isolation and characterization of BM-MSC. Under complete aseptic conditions, bone marrow was harvested from the tibia and femur of 8-week-old male white Wistar rats by flushing using low glucose Dulbecco's Modified Eagle's Medium (DMEM) [Gibco, Gainesville, MD, USA], supplemented with $10 \%$ fetal bovine serum [FBS, GIBCO/ /BRL]. The isolated cells were cultured in a growth medium containing $1 \%$ penicillin/streptomycin [GIBCO] and incubated in $5 \%$ humidified $\mathrm{CO}_{2}$ at $37^{\circ} \mathrm{C}$ for about 2 weeks as a primary culture or when large colonies appeared. At $80 \%$ confluence, the cells were washed with PBS. The cells were trypsinized with $0.25 \%$ trypsin in $1 \mathrm{mM}$ ethylene-diaminetetraacetic acid for $5 \mathrm{~min}$ at $37^{\circ} \mathrm{C}$. After centrifugation, cells were resuspended with serum-supplemented medium (10\% FBS) and incubated in $50 \mathrm{~cm}^{2}$ culture Falcon flask. The subculture passage was done till the $3^{\text {rd }}$ passage to expand the cell population [13]. BM-MSCs were characterized by their adhesiveness and fusiform shape and with flow cytometry (CYTOMICS FC 500, Beckman Coulter, Champaign, IL, USA) identification of the mesenchymal stem cells surface markers [14]. The BM-MSCs were positive for CD90 and CD105 and negative for CD45 and CD34.

Labeling of BM-MSc with PKH26 fluorescent linker dye. The MSCs were harvested during the $3^{\text {rd }}$ passage and labeled with PKH26, a lipophilic red fluorescent linker dye (Cat. \# MINI26, Sigma Aldrich, St. Louis, MO, USA). It was used to localize the injected stem cells in the uterine tissue. The BMMSCs were centrifuged and washed 2 times in serum-free medium and pelleted. Then, they were incubated for $1 \mathrm{~h}(5 \%$ $\mathrm{CO}_{2}$ at $37^{\circ} \mathrm{C}$ ) in a cocktail solution consisting of serum-free 
DMEM (83.3\% vol/vol) and $1.1 \%$ of PKH26 dye solution in diluent ad $100 \%$. The staining was stopped according to the manufacturer's recommendations (SigmaAldrich). The PKH26-labeled BM-MSCs were examined using phase-contrast Olympus CKX53 fluorescent microscope (Olympus, Tokyo, Japan) and maintained in the growth medium for the following experiment to be injected intravenously into the rat tail vein [15] of the animals in the group GpIII of rats (AS rats BMSCs-treated).

Vaginal smear examination. Vaginal smears were obtained with a micropipette [16]. The tip of the micropipette was filled with a small amount of saline (1-2 drops), then inserted into the vagina of the female rat. The vagina was flushed 2-3 times with the saline and then the fluid was placed onto a glass slide. The fluid was equally distributed onto the slides and was spread out. Samples were left to dry without fixation and then stained with $1 \%$ crystal violet. Determination of the phases of the estrous cycle was made using a light microscope.

Biological samples' collection. The rats were euthanized using an intraperitoneal injection of thiopental sodium-500 (90 mg $/ \mathrm{kg}$; EPICO, 10th of Ramadan City, Egypt). Specimens of mid-portion of the Rt uterine horn were collected, fixed in $10 \%$ formol saline, and processed into paraffin blocks. Serial sections, 5-7 $\mu \mathrm{m}$ thickness, were obtained and subjected to fluorescent, histological and immunohistochemical studies. The stained sections were examined by expertized histologists using light microscope connected to Olympus camera (BX-50 f4, Olympus Optical Co. Ltd., Japan). To detect the homing of the PKH26-labeled BM-MSCs in the uterine tissue, a reflected light fluorescent microscope (Olympus) was used.

Histological studies. Hematoxylin and eosin (H\&E) staining was used for general morphological analysis [17] and Masson's trichrome staining to elucidate collagen deposition [18].

Immunohistochemistry. Anti-PCNA mouse monoclonal antibody (Ab) (Cat. \# MS106P Thermo Scientific Laboratories, Fermont, CA, USA) was used as a standard marker for the proliferating cells and anti-VEGF rabbit polyclonal $\mathrm{Ab}$ [Boster Biological Technology, Pleasanton, CA, USA, Cat. \# PA1080] was applied to demonstrate neovascularization in the endometrium [19].

Anti-NF- $\kappa$ B rabbit polyclonal Ab (Cat. \# PA1669; Boster Biological Technology) was used to detect this transcription factor that links the pathogenic signals and cellular danger signals.

Immunohistochemistry required pre-treatment by boiling the deparaffinized sections in $10 \mathrm{mM}$ citrate buffer (Cat. \# 005000) pH 6 for 10 min for the antigen retrieval.
The sections were allowed to cool in RT for 20 min then endogenous peroxidase activity was blocked by hydrogen peroxide and the sections were then rinsed in PBS. Incubation with the primary $\mathrm{Ab}$ was performed for $1 \mathrm{~h}$. The biotinylated secondary Ab (Cat. \# 62-9520; Life Technologies) was applied. Immunostaining was completed by the use of Ultravision One Detection System (Cat. \# TL-060-HLJ; Life Technologies). Adding of diaminobenzidine (DAB) solution resulted in a brown-colored precipitate. Counter-staining was performed using Meyer's hematoxylin (Cat. \# TA-060-MH; Life Technologies) to visualize the nuclei. Negative controls were prepared using the same steps with omitting the primary $\mathrm{Ab}$.

Morphometry. Data were obtained using "Leica Qwin-500 C" image analyzer (Cambridge, England) in the Histology Department, Faculty of Medicine, Cairo University. The slides were examined under the light microscope and ten non-overlapping randomly selected low power fields $(\times 100)$ / /slide were examined to measure: (i) the thickness of endometrium and number of uterine glands in the H\&E stained sections (using interactive measuring menu); (ii) relative area of collagen deposition in Masson's trichrome stained sections (measured using the color detect menu with a standard measuring frame with an area $116946.91 \mu \mathrm{m}^{2}$ ); (iii) the number of PCNA positive (+ve) immunostained cells; (iv) relative area of VEGF and NF- $\kappa$ B immunostained cells (areas of positivity were measured using the color detect menu with a standard measuring frame with an area $116946.91 \mu \mathrm{m}^{2}$ ).

Statistical analysis. The obtained measurements were analyzed using the SPSS software version 16 (SPSS Inc., Chicago, IL, USA). Comparisons between different groups were done using one-way analysis of variance (ANOVA) test followed by a post-hoc Tukey test. Quantitative data were summarized as means and standard deviations (SD). The p-value of less than 0.05 was considered statistically significant.

\section{Results}

\section{Clinical observation}

No death was registered in the experimental animals nor changes in their conduct of the water and food consumption.

\section{Vaginal smear morphology}

The estrous cycle was assessed by the daily morning vaginal smears examination. The estrus phase was identified by the presence of predominant cornified squamous epithelial cells with no apparent nuclei. Cells had an angular appearance and present in densely packed clusters (Fig. 1). 


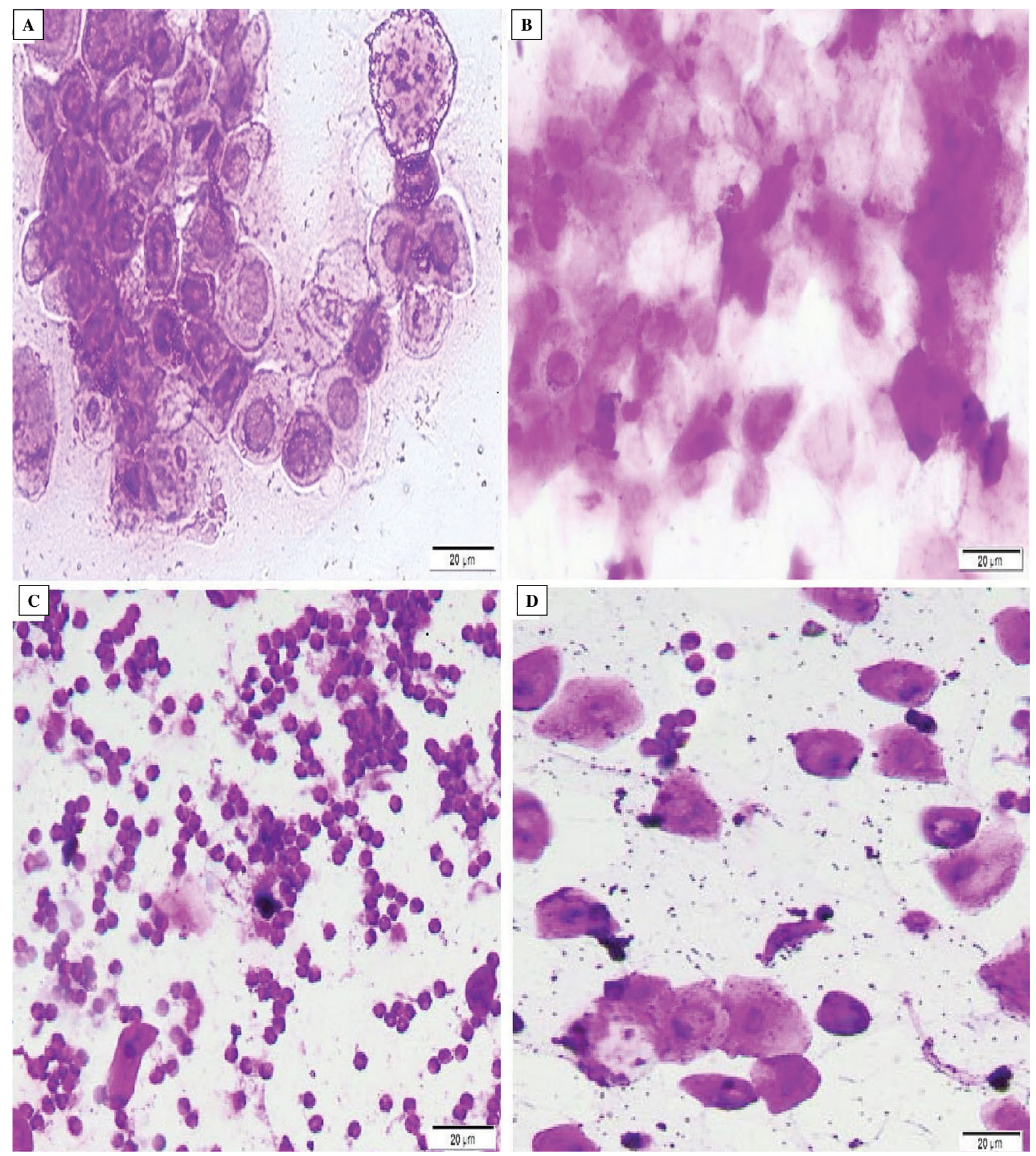

Figure 1. Photomicrographs of crystal violet stained vaginal smear showing different phases of estrous cycle of the rat. A. Proestrus phase: rounded, well-formed nucleated epithelial cells with darkly stained nuclei present in clusters. B. Estrus phase: predominant cornified squamous epithelial cells with no apparent nuclei, present in densely packed clusters. C. Metestrus phase: the predominant cells are small darkly stained leukocytes with few scattered cornified squamous epithelial cells. D. Diestrus phase: the predominant cells are leukocytes. Nucleated epithelial cells also present. Magnification: $400 \times$.

\section{Characteristics of the BM-MSCs}

Fluorescent microscopic examination of the unstained uterine sections of GpIII (AS rats BMSCs-treated group) revealed red fluorescent cells distributed within the regenerated endometrium (Fig. 2), thus confirming that injected BM-MSCs labeled with $\mathrm{PKH} 26$ fluorescent dye homed into the uterus. 


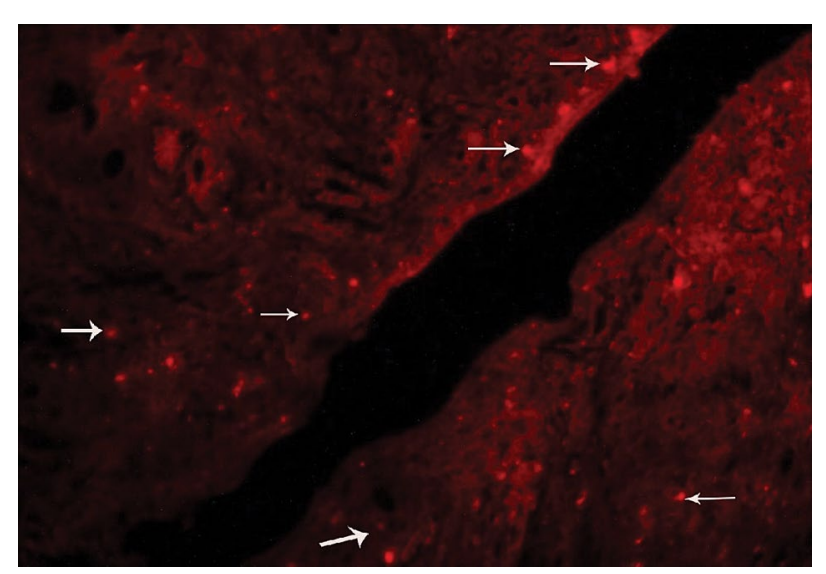

Figure 2. Photomicrograph of a section rat uterus of GpIII group. (White-arrows): red fluorescent cells. (PKH26 fluorescence, $200 \times)$.

\section{Morphological observations}

Effects of TCA instillation on the morphology of rat uterus

Uteri of the control rats in the estrous cycle showed thick endometrium harboring numerous uterine glands, intact surface, and glandular epithelium with highly cellular CT stroma containing numerous blood vessels (Fig. 3A). These uterine changes define the onset of the estrus phase. The model group exhibited thin endometrium, few distorted glands, and little cellularity of the fibrous stroma. In GpIV, the endometrium was thin with few glands and discontinuous surface epithelium (Fig. 3B). Masson's trichrome-stained uterine sections of $\mathrm{GpI}$ showed fine collagen fibers (Fig. 4A). In GpII, marked collagen deposition in the lamina propria of the thin endometrium was observed

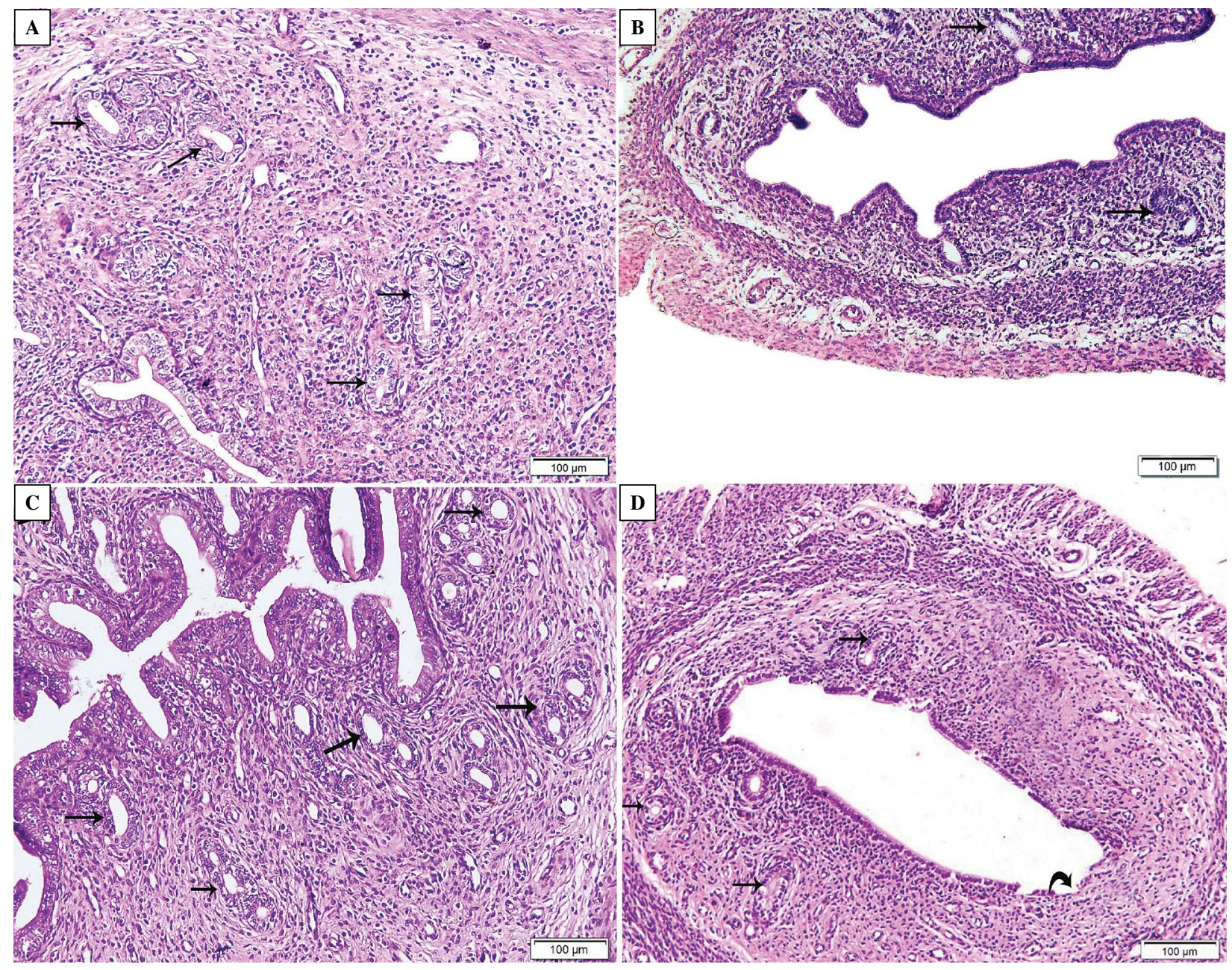

Figure 3. H\&E-stained rat uterine sections. A. GpI (control): thick endometrium, numerous uterine glands (arrows) occupying most of the endometrial thickness and lined with simple cuboidal cells with vesicular nuclei and vacuolated cytoplasm, and intact surface epithelium. The lamina propria contains highly cellular connective tissue (CT) with stromal cells exhibiting vesicular nuclei and many leukocytic infiltrations. B. GpII (AS model): pronounced thinning of the endometrium with few uterine glands (arrow). C. GpIII (AS rats BMSCs-treated): preserved endometrial thickness, numerous uterine glands (arrows), intact surface epithelium and the stroma is highly cellular CT with many leukocytic infiltrations. D. GpIV (BMSCs-untreated AS rat): thin endometrium, few endometrial glands (arrow), and discontinuous surface epithelium (curved arrow). Magnification: 100×. 

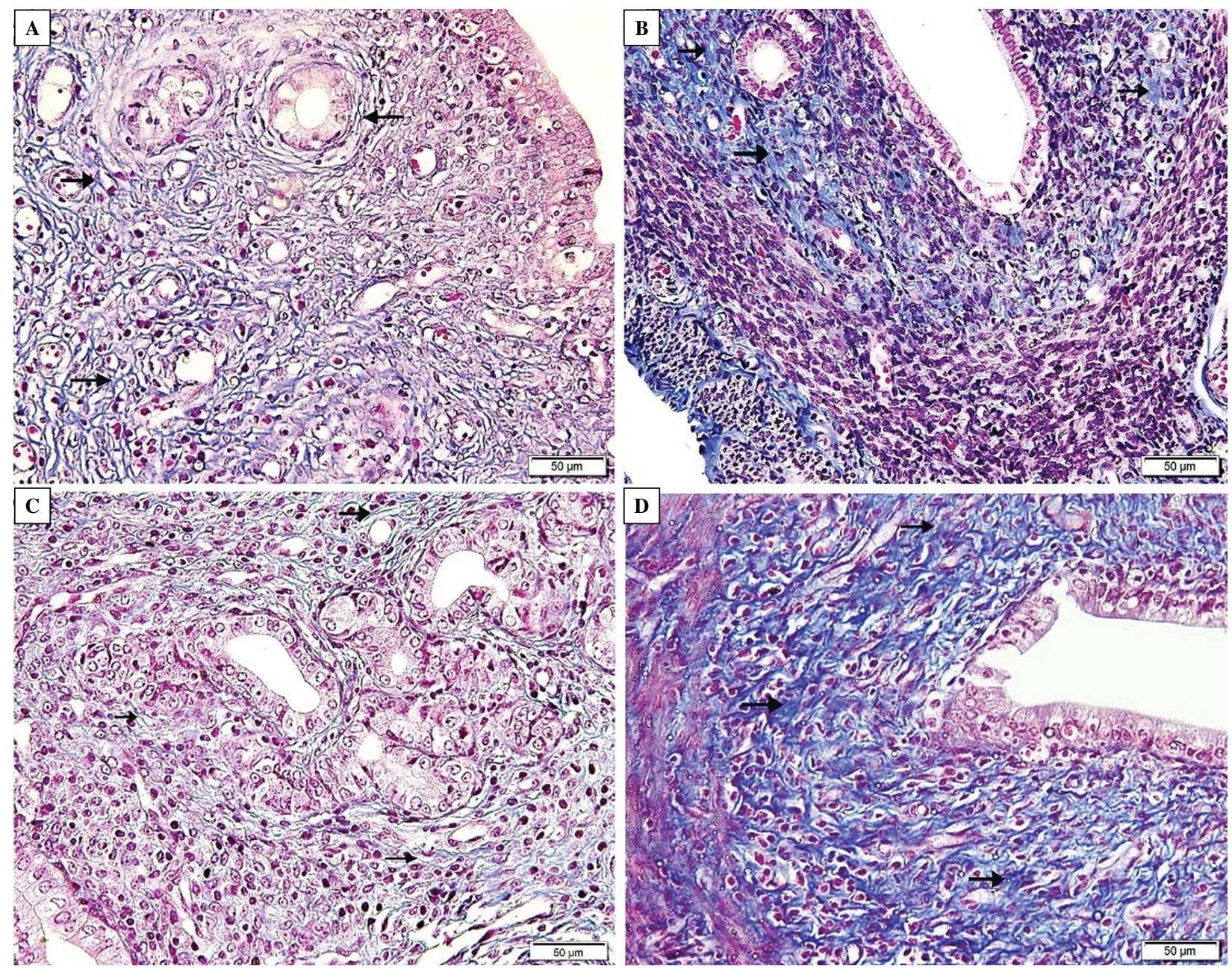

Figure 4. Uterine sections stained by Massson's trichrome method. A. GpI (control): fine collagen fibers (arrow). B. GpII (AS model): coarse collagen deposition in the lamina propria. C. GpIII (AS rats BMSCs-treated): fine collagen fibers between stromal cells and surrounding the glands. (D) GpIV (untreated AS): coarse collagen deposition in the endometrial stroma. Magnification: $100 \times$.

(Fig. 4B). In GpIV, obvious coarse collagen deposition in the stroma of the endometrium was noted (Fig. 4D).

\section{Effects of BM-MSCs on the features of AS endometrium}

Regenerated endometrium was noted in GpIII that showed thick endometrium, numerous uterine glands, intact surface epithelium and highly cellular stroma with numerous leukocytic infiltrations (Fig. 3C). Besides, fine collagen fibers between the stromal cells, and surrounding the glands (Fig. 4C).

\section{Immunohistochemical stainings}

Immunohistochemical detection of PCNA in uterine sections revealed in GpI strong widespread nuclear PCNA immunoreactivity (-Ir). GpII illustrated weak PCNA-Ir in some cells of the surface epithelium, the obliterated glands, and the stromal cells. GpIII displayed widespread nuclear PCNA-Ir in the surface epithelium, the uterine glands and the stromal cells.
In GpIV nuclear immunostaining was detected in the surface epithelium and some stromal cells (Figs. $5 \mathrm{~A}-\mathrm{D}$, respectively).

Immunohistochemical detection of VEGF in uterine sections of GpI rats revealed widespread prominent positive immunostaining was observed. GpII showed few positive VEGF immunostaining in the stroma. In GpIII, diffuse positive immunostaining was detected in the surface epithelium, uterine glands, stromal cells, and the endothelium. GpIV showed some positive VEGF immunostaining in the surface epithelium and the stroma. (Figs. 5E-H, respectively).

Immunohistochemical detection of $\mathrm{NF}-\kappa \mathrm{B}$ revealed in $\mathrm{GpI}$ moderate positive immunostaining. In uterus of GpII rats, strong NF- $\kappa$ B-Ir was observed in the cytoplasm and cell nuclei of the surface epithelium, endometrial glands and almost all stromal cells. In GpIII, moderate NF- $\kappa$ B-Ir was demonstrated in the cytoplasm of the surface epithelium and in the uterine 


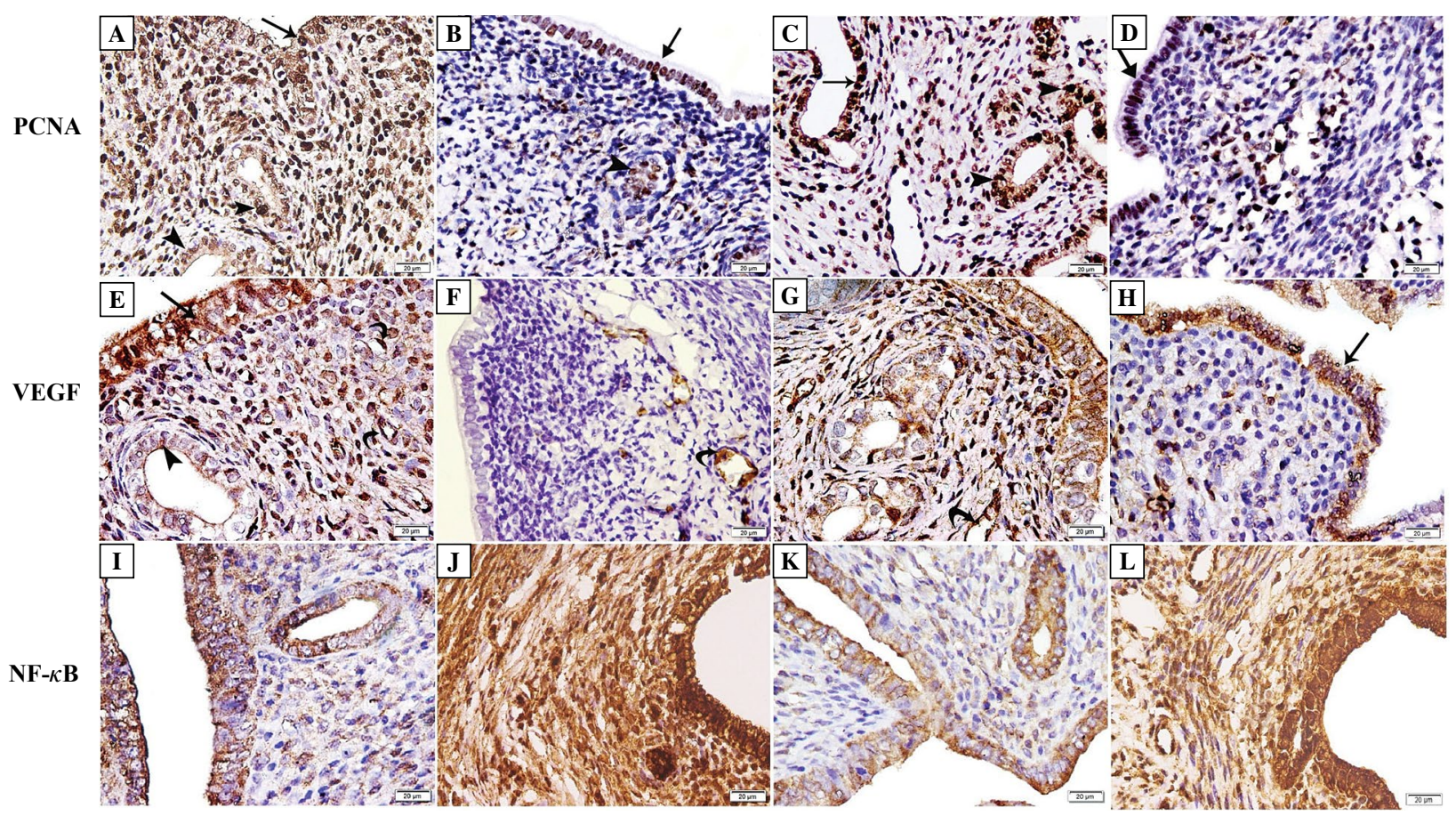

Figure 5. Immunoexpression of PCNA, VEGF, and NF- $\kappa$ B in the uterine section. PCNA immunohistochemical staining: (A) GpI: strong nuclear PCNA immunostaining in the surface epithelium (arrow), endometrial glands (arrowhead), and many stromal cells. (B) GpII: weak nuclear immunostaining. (C) GpIII: widespread nuclear immunostaining. (D) GpIV: weak nuclear immunostaining. VEGF immunohistochemical staining: (E) GpI: widespread prominent +ve immunostaining in the epithelium lining the lumen (arrow), the uterine glands (arrowheads), the stromal cells, and the blood vessels (curved arrow). (F) GpII: minimal +ve immunostaining detected in few stromal cells and in the endothelium (curved arrow). (G) GpIII: diffuse +ve immunostaining. (H) GpIV: some +ve immunostained cells in the surface epithelium (arrow) and in the stromal cells. NF- $\kappa$ B immunohistochemical staining: (I) GpI: mild cytoplasmic +ve immunostaining. (J) GpII: strong diffuse +ve cytoplasmic and nuclear immunostaining. (K) GpIII: mild cytoplasmic + ve immunostaining. (L) GpIV: strong diffuse +ve cytoplasmic and nuclear immunostaining. Magnification: $400 \times$.

Table 1. The mean $( \pm$ SD) of endometrial thickness, number of the uterine glands, area $\%$ of collagen deposition, number of PCNA + ve cells, area \% of VEGF immunostaining, and area $\%$ of NF- $\kappa$ B immunostaining in the control and the experimental groups

\begin{tabular}{|l|c|c|c|c|}
\hline Parameters/Groups & Gp I & Gp II & Gp III & Gp IV \\
\hline Endometrial thickness $[\mu \mathrm{m}]$ & $406 \pm 19.3$ & $93.7 \pm 15.4^{* \#}$ & $390.4 \pm 11.3$ & $97.5 \pm 24.2^{* \#}$ \\
\hline Number of uterine glands & $8.7 \pm 1.9$ & $0.9 \pm 0.7^{* \#}$ & $8.5 \pm 1.1$ & $3.1 \pm 0.7^{* \# \dagger}$ \\
\hline Area \% of collagen deposition & $20.9 \pm 3.8$ & $42.2 \pm 4^{* \#}$ & $19.8 \pm 4.1$ & $59.1 \pm 3.5^{* \# \dagger}$ \\
\hline Number of PCNA + ve cells & $54.1 \pm 5.4$ & $19.5 \pm 3.4^{* \#}$ & $45.7 \pm 2.8$ & $30.6 \pm 2.4^{* \# \dagger}$ \\
\hline Area \% of VEGF immunostaining & $59.4 \pm 3.6$ & $22 \pm 3.7^{* \#}$ & $42.5 \pm 3.2$ & $33.9 \pm 7.3^{* \#+}$ \\
\hline Area \% of NF- $\kappa$ B immunostaining & $20.2 \pm 7.6$ & $50.5 \pm 3.2^{* \#}$ & $22.7 \pm 7.3$ & $52.2 \pm 1.1^{* \#}$ \\
\hline
\end{tabular}

*Significant $(\mathrm{p}<0.05)$ as compared to GpI, ${ }^{\circ}$ Significant as compared to GpII, ${ }^{\#}$ Significant as compared to GpIII.

gland. GpIV displayed widespread positive cytoplasmic and nuclear NF- $\kappa \mathrm{B}$ immunostaining (Fig. 5I-L).

\section{Morphometric Study}

The data of the morphometric measurements are presented in Table 1.
Regeneration of the endometrium

and the uterine glands by BM-MSC

The mean thickness of the endometrium and the mean number of the endometrial glands in the BMSC-treated group showed significant $(p<0.000$ and $p<0.001$ ) increase, respectively, as compared 
to GpII and GpIV groups, with a non-significant difference as compared to GpI group, thus denoting regenerative capacity of the stem cells therapy.

\section{The antifibrotic effect of BM-MSCs}

The mean area $\%$ of collagen deposition was substantially increased in the endometrium of Asherman model rats $(\mathrm{p}<0.000)$ and recovery groups $(\mathrm{p}<0.002)$ as compared to the control. Meanwhile, it was significantly $(p<0.001)$ decreased in GpIII with the treatment of BM-MSCs as compared to GpII indicating the antifibrotic effect of BM-MSC.

\section{Effect of BM-MSCs on the immunoexpression of PCNA, VEGF and NF- $\kappa B$}

In the uterus of Asherman model and the recovery group rats, the expression of PCNA and VEGF was significantly ( $p<0.001)$ decreased as compared to GpI. Also, the mean area \% of NF- $\kappa$ B immunostaining was significantly $(\mathrm{p}<0.001)$ increased in both groups. Stem cell therapy substantially increased the expression of PCNA and VAGF in the endometrium and significantly decreased the expression of NF- $\kappa \mathrm{B}$ (Table 10).

\section{Discussion}

Asherman syndrome is referred to intrauterine synechiae due to injury of the endometrial basal layer accompanied by thin endometrium [19]. Surgery serves only to divide the adhesions within the cavity, but can do very little about the endometrial regeneration and adhesion recurrence [20]. Therefore, we tried to use bone marrow-derived mesenchymal stem cells for the regeneration of the endometrium in the experimental intrauterine synechia AS model. After the induction of AS, euthanization was performed in the estrus phase of the $3^{\text {rd }}$ cycle to assess the establishment of the modeling based on the study of Cocuzza et al. and Kilic et al., [10,11]. Asherman syndrome is known to be one of the causes of thin endometrium. Defective uterine perfusion was assumed to be the reason for the atrophied endometrium due to limited exposure to the circulating hormones [21]. The endometrial thickness is mandatory for the successful implantation and pregnancy compared to low receptivity and pregnancy rate in case of thin endometrium [22].

The stroma of rats in GpII (AS group) showed little cellularity, few blood vessels and obvious fibrosis with substantial increase in the mean area $\%$ of the collagen deposition as compared to GpI (control) and Gp III (AS rats BMSCs-treated). Generally, fibrosis is assumed to occur as a result of interaction between the inflammatory and the fibrotic cytokines. IL- 6 acts as a pro-inflammatory cytokine that is secreted mainly by inflammatory cells as macrophages and stimulates the immune response. It contributes to the development of fibrosis by modulating TGF $\beta$ signaling and stimulating the proliferation of fibroblasts, as well as the collagen production [23].

Intravenous injection of BM-MSCs revealed regenerated (increased) endometrial thickness and numerous uterine glands with intact surface and glandular epithelium, as compared to the control group, indicating regaining the normal estrous cyclic changes. Intravenous injection of stem cells has been documented to be effective in experimental studies [24, 25]. As compared to local tissue injection of stem cells, systemic administration is more easy applicable method that empowers the clinical use of this therapy [24]. Moreover, homing of the injected stem cells to the injured tissue has been documented in previous studies $[6,14]$ and confirmed in our present work by localization of the PKH26-labeled stem cells in the uterine tissue. Jing et al. [12] referred this regenerative uterine capacity to the therapeutic effect of BM-MSCs by exerting protective actions against cell damage. Exogenous BM-MSCs could home to damaged tissues where they are releasing trophic factors rather than forming engraftment. They can produce high amounts of anti-inflammatory cytokines and growth factors, and possess immunomodulatory properties [26]. A relevant phenomenon is that BM-MSCs up-regulate the anti-inflammatory cytokines, such as fibroblast growth factor- $\beta$ and IL-6, and down-regulate the pro-inflammatory cytokines, such as TNF- $\alpha$ and IL-1 $\beta$ [12].

The lamina propria of the uterine sections in GpIII (BM-MSC-treated AS rats) contained highly cellular CT with numerous blood vessels and fine collagen fibers with the mean area $\%$ of collagen deposition comparable to the control rats. These results are going parallel with Sabry et al. [6] who documented in an induced-endometrial fibrosis rat model the antifibrotic effect of stem cells by significant decrease in IL-1 gene expression. IL-1gene expression was accused in the development of fibrosis. Similarly, systemic infusion of BM-MSCs in cutaneous radiation induced fibrosis rat model reduced skin contracture, thickening, collagen deposition and decreased expression of IL-1 in their radiated skin [27]. Ebrahim et al. [28], in an intrauterine adhesion female rat model, showed that MSCs secreted exosomes or vesicles, which are enriched in the extracellular environment. These vesicles have been considered as vital mediators of cellular communication and may regulate various physiological and pathological processes by transferring membrane proteins, mRNAs, and miRNAs to recipient cell [29]. Recent studies have demonstrated that vesicles can act during different stages of the inflammatory re- 
sponse by transporting bioactive factors and suppress the inflammation in different animal models to restore the physiological status [30, 31].

The endometrial regeneration in AS rats induced by BM-MSC administration seems to be specifically caused by this procedure since uterine sections of rats from GpIV (untreated AS) showed thin endometrium with discontinuous surface epithelium. Few endometrial glands were seen scattered in the fibrosed endometrium suggesting poor proliferation by the tissue stem cells.

It is well known that PCNA has a crucial role in accurate DNA duplication [32]. Rats in GpII and GpIV groups demonstrated weak PCNA immunostaining in the endometrium. Meanwhile, AS BMSCs-treated rats displayed significant increase in PCNA immunostainig in the endometrial tissue indicating preservation of the proliferative capacity of the endometrial cells [33]. The bone marrow progenitor cells were documented to improve the proliferation of different injured tissues [34, 35]. Stem cell effects could be through direct homing to the injured tissue and repairing the endometrium through self-renewal, proliferation, and differentiation. In addition, mesenchymal stem cell has a paracrine effect on the surrounding cell to increase the efficacy of tissue repair [36].

Asherman syndrome is frequently associated with defective blood supply to the endometrium with subsequent impaired hormonal delivery to the endometrium resulting in thin endometrium [37]. In the present work, rats in GpII and GpIV groups displayed significant decrease in VEGF expression in the uterine tissue. In the fibrotic stroma of the endometrium of AS, thin-walled telangiectatic blood vessels are thought to cause impaired blood perfusion and tissue atrophy [38]. However, BMSC-treated AS-rats demonstrated significant diffuse VEGF immunostaining in the epithelium, stromal cells and endothelial cells indicating improved angiogenesis of the regenerated endometrium. Ebrahim et al. [28] postulated that the ability of MSC to repair uterine scars was associated with up-regulation of VEGF and down-regulation of collagen type I expression, in addition to promoting the regeneration of the endometrium, myometrium, and blood vessels in the uterine scars.

The NF- $\kappa \mathrm{B}$ signaling pathway is considered a spark for the inflammatory process through its triggering role in the up-regulation of various pro-inflammatory genes encoding chemokines, cytokines, and adhesion molecules $[39,40]$. NF- $\kappa$ B activation was documented in the pathogenesis of various inflammatory diseases [35]. In AS, the intrauterine adhesions are associated with increased level of the inflammatory cytokines as
TGF- $\beta$, TNF- $\alpha$, IL- 1 , and IL- 18 that crosstalk with the NF-kB pathway [41]. In the present work, a significant increase in NF- $\kappa \mathrm{B}$ immunostaining was detected in rats with the induced AS and the recovery group. In agreement with previous study [2], the importance of NF- $\kappa \mathrm{B}$ was confirmed in the pathogenesis of human AS and animal models and suggested its role as a potential diagnostic marker and therapeutic target in the treatment of intrauterine adhesions. The immunomodulatory role and anti-inflammatory effect of stem cells were documented [2]. In a recent study, MSC-conditioned media reduced the inflammation in endotoxin-induced acute lung injury through inhibition of the activity of NF- $\kappa$ B and increasing the interleukin- 6 and macrophage inflammatory protein level [42].

Although stem cell therapy was shown to be safe and well-tolerated in the clinical trials [43], the risk of tumorigenicity must always be evaluated with long term follow up, and during the culture- expansion stage before any therapeutic infusion [44].

In summary, application of BM-MSCs effectively promoted the endometrial regeneration in Asherman syndrome rat model through improving the proliferative capacity of the uterine tissue, inducing angiogenesis, and modulating the inflammatory process and the fibrosis.

\section{Conflict of interest}

All authors confirm that there is no conflict of interest.

\section{References}

1. Dreisler E, Kjer JJ. Asherman's syndrome: current perspectives on diagnosis and management. Int $\mathrm{J}$ Womens Health. 2019; 11: 191-198, doi: 10.2147/IJWH.S165474, indexed in Pubmed: 30936754.

2. Wang X, Ma N, Sun Q, et al. Elevated NF- $\kappa$ B signaling in Asherman syndrome patients and animal models. Oncotarget. 2017; 8(9): 15399-15406, doi: 10.18632/oncotarget.14853, indexed in Pubmed: 28148903.

3. March CM. Management of Asherman's syndrome. Reprod Biomed Online. 2011; 23(1): 63-76, doi: 10.1016/j. rbmo.2010.11.018, indexed in Pubmed: 21549641.

4. Wang J, Ju B, Pan C, et al. Application of Bone Marrow-Derived Mesenchymal Stem Cells in the Treatment of Intrauterine Adhesions in Rats. Cell Physiol Biochem. 2016; 39(4): 1553-1560, doi: 10.1159/000447857, indexed in Pubmed: 27614987.

5. Conforti A, Alviggi C, Mollo A, et al. The management of Asherman syndrome: a review of literature. Reprod Biol Endocrinol. 2013; 11: 118, doi: 10.1186/1477-7827-11-118, indexed in Pubmed: 24373209.

6. Sabry D, Mostafa A, Marzouk S, et al. Neupogen and mesenchymal stem cells are the novel therapeutic agents in regeneration of induced endometrial fibrosis in experimental rats. Biosci Rep. 2017; 37(5), doi: 10.1042/BSR20170794, indexed in Pubmed: 28883083. 
7. Tang L, Chen Yu, Pei F, et al. Lithium Chloride Modulates Adipogenesis and Osteogenesis of Human Bone Marrow-Derived Mesenchymal Stem Cells. Cell Physiol Biochem. 2015; 37(1): 143-152, doi: 10.1159/000430340, indexed in Pubmed: 26303458.

8. Golpanian S, Wolf A, Hatzistergos KE, et al. Rebuilding the Damaged Heart: Mesenchymal Stem Cells, Cell-Based Therapy, and Engineered Heart Tissue. Physiol Rev. 2016; 96(3): 1127-1168, doi: 10.1152/physrev.00019.2015, indexed in Pubmed: 27335447.

9. Santamaria X, Cabanillas S, Cervelló I, et al. Autologous cell therapy with CD133+ bone marrow-derived stem cells for refractory Asherman's syndrome and endometrial atrophy: a pilot cohort study. Hum Reprod. 2016; 31(5): 1087-1096, doi: 10.1093/humrep/dew042, indexed in Pubmed: 27005892.

10. Cocuzza MA, Cocuzza M, Maciel GAR, et al. Development of an animal model for endometrial ablation using trichloroacetic acid. Fertil Steril. 2011; 95(7): 2418-2421, doi: 10.1016/j. fertnstert.2011.03.064, indexed in Pubmed: 21497335.

11. Kilic S, Yuksel B, Pinarli F, et al. Effect of stem cell application on Asherman syndrome, an experimental rat model. J Assist Reprod Genet. 2014; 31(8): 975-982, doi: 10.1007/ s10815-014-0268-2, indexed in Pubmed: 24974357.

12. Jing Z, Qiong Z, Yonggang W, et al. Rat bone marrow mesenchymal stem cells improve regeneration of thin endometrium in rat. Fertil Steril. 2014; 101(2): 587-594, doi: 10.1016/j. fertnstert.2013.10.053, indexed in Pubmed: 24355044.

13. Alhadlaq A, Mao JJ. Mesenchymal stem cells: isolation and therapeutics. Stem Cells Dev. 2004; 13(4): 436-448, doi: 10.1089/scd.2004.13.436, indexed in Pubmed: 15345137.

14. Mohi El-Din MM, Rashed LA, Mahmoud Haridy MA, et al. Impact of bone marrow-derived mesenchymal stem cells on remodeling the lung injury induced by lipopolysaccharides in mice. Future Sci OA. 2017; 3(1): FSO162, doi: 10.4155/fsoa2016-0036, indexed in Pubmed: 28344826.

15. MT AA, MA, W. Mesenchymal Stem Cells Therapy in Acute Renal Failure: Possible Role of Hepatocyte Growth Factor. J Stem Cell Res Ther. 2011; 01(03), doi: 10.4172/21577633.1000109.

16. Yener T, Turkkani Tunc A, Aslan H, et al. Determination of oestrous cycle of the rats by direct examination: how reliable? Anat Histol Embryol. 2007; 36(1): 75-77, doi: 10.1111/j.14390264.2006.00743.x, indexed in Pubmed: 17266672.

17. Bancroft JD, Gamble M. The hematoxylin and eosin. In: Theory and Practice of Histological Techniques, 6th ed. London: Churchill Livingstone. ; 2008: 121-134.

18. Bancroft JD, Gamble M. 2008. Connective tissue and stain. In: Theory and Practice of Histological Techniques, 6th ed. London: Churchill Livingstone. ; 2008: 147-150.

19. Smikle C, Khetarpal S. Asherman Syndrome. Stat Pearls [Internet], Treasure Island (FL), StatPearls Publishing. ; 2020.

20. Guo EJ, Chung JP, Poon LC, et al. Reproductive outcomes after surgical treatment of asherman syndrome: A systematic review. Best Pract Res Clin Obstet Gynaecol. 2019; 59: 98-114, doi: 10.1016/j.bpobgyn.2018.12.009, indexed in Pubmed: 30713131.

21. Senturk LM, Erel CT. Thin endometrium in assisted reproductive technology. Curr Opin Obstet Gynecol. 2008; 20(3): 221-228, doi: 10.1097/GCO.0b013e328302143c, indexed in Pubmed: 18460935.

22. Eftekhar M, Tabibnejad N, Tabatabaie A. The thin endometrium in assisted reproductive technology: An ongoing challenge. Middle East Fertility Society Journal. 2018; 23(1): 1-7, doi: 10.1016/j.mefs.2017.12.006.
23. Luckett-Chastain LR, Gallucci RM. Interleukin (IL)-6 modulates transforming growth factor-beta expression in skin and dermal fibroblasts from IL-6-deficient mice. Br J Dermatol. 2009; 161(2): 237-248, doi: 10.1111/j.1365-2133.2009.09215.x, indexed in Pubmed: 19438433.

24. Ramalho BD, Almeida FM, Sales CM, et al. Injection of bone marrow mesenchymal stem cells by intravenous or intraperitoneal routes is a viable alternative to spinal cord injury treatment in mice. Neural Regen Res. 2018; 13(6): 1046-1053, doi: 10.4103/1673-5374.233448, indexed in Pubmed: 29926832.

25. Zickri MB, Fadl SG, Metwally HG. Comparative Study between Intravenous and Intraperitoneal Stem Cell Therapy in Amiodarone Induced Lung Injury in Rat. Int J Stem Cells. 2014; 7(1): 1-11, doi: 10.15283/ijsc.2014.7.1.1, indexed in Pubmed: 24921022.

26. Fu Y, Karbaat L, Wu L, et al. Trophic Effects of Mesenchymal Stem Cells in Tissue Regeneration. Tissue Eng Part B Rev. 2017; 23(6): 515-528, doi: 10.1089/ten.TEB.2016.0365, indexed in Pubmed: 28490258.

27. Horton JA, Hudak KE, Chung EJ, et al. Mesenchymal stem cells inhibit cutaneous radiation-induced fibrosis by suppressing chronic inflammation. Stem Cells. 2013; 31(10): 2231-2241, doi: 10.1002/stem.1483, indexed in Pubmed: 23897677.

28. Ebrahim N, Mostafa O, El Dosoky RE, et al. Human mesenchymal stem cell-derived extracellular vesicles/estrogen combined therapy safely ameliorates experimentally induced intrauterine adhesions in a female rat model. Stem Cell Res Ther. 2018; 9(1): 175, doi: 10.1186/s13287-018-0924-z, indexed in Pubmed: 29954457.

29. Toh WS, Lai RC, Zhang B, et al. MSC exosome works through a protein-based mechanism of action. Biochem Soc Trans. 2018; 46(4): 843-853, doi: 10.1042/BST20180079, indexed in Pubmed: 29986939.

30. Zhang S, Teo KY, Chuah SJ, et al. MSC exosomes alleviate temporomandibular joint osteoarthritis by attenuating inflammation and restoring matrix homeostasis. Biomaterials. 2019; 200: 35-47, doi: 10.1016/j.biomaterials.2019.02.006, indexed in Pubmed: 30771585.

31. Omar A, Aboulkhair A. Do microvesicles derived from adipose mesenchymal stem cells have a therapeutic potential on Escherichia coli lipopolysaccharides-induced sepsis in Zona fasciculata of adult male albino rats? A histological study. EJH. 2018; 41(2): 204-219, doi: 10.21608/ejh.2018.13843.

32. Kang MS, Ryu E, Lee SW, et al. Regulation of PCNA cycling on replicating DNA by RFC and RFC-like complexes. Nat Commun. 2019; 10(1): 2420, doi: 10.1038/s41467-019-10376-w, indexed in Pubmed: 31160570.

33. Li B, Zhang Q, Sun J, et al. Human amniotic epithelial cells improve fertility in an intrauterine adhesion mouse model. Stem Cell Res Ther. 2019; 10(1): 257, doi: 10.1186/s13287019-1368-9, indexed in Pubmed: 31412924.

34. Shawky LM, El Bana EA, Morsi AA. Stem cells and metformin synergistically promote healing in experimentally induced cutaneous wound injury in diabetic rats. Folia Histochem Cytobiol. 2019; 57(3): 127-138, doi: 10.5603/FHC.a2019.0014, indexed in Pubmed: 31489604.

35. Mohsen RO, Halawa AM, Hassan R. Role of bone marrow-derived stem cells versus insulin on filiform and fungiform papillae of diabetic albino rats (light, fluorescent and scanning electron microscopic study). Acta Histochem. 2019; 121(7): 812-822, doi: 10.1016/j.acthis.2019.07.007, indexed in Pubmed: 31358295.

36. Fu X, Liu Ge, Halim A, et al. Mesenchymal Stem Cell Migration and Tissue Repair. Cells. 2019; 8(8), doi: 10.3390/ cells8080784, indexed in Pubmed: 31357692. 
37. Miwa I, Tamura H, Takasaki A, et al. Pathophysiologic features of „thin“ endometrium. Fertil Steril. 2009; 91(4): 998-1004, doi: 10.1016/j.fertnstert.2008.01.029, indexed in Pubmed: 18328483.

38. Santamaria X, Isaacson K, Simón C. Asherman‘s Syndrome: it may not be all our fault. Hum Reprod. 2018; 33(8): 1374-1380, doi: 10.1093/humrep/dey232, indexed in Pubmed: 31986212.

39. Lawrence T. The nuclear factor NF-kappaB pathway in inflammation. Cold Spring Harb Perspect Biol. 2009; 1(6): a001651, doi: 10.1101/cshperspect.a001651, indexed in Pubmed: 20457564.

40. Liu T, Zhang L, Joo D, et al. NF- $\kappa$ B signaling in inflammation. Signal Transduct Target Ther. 2017; 2, doi: 10.1038/ sigtrans.2017.23, indexed in Pubmed: 29158945.

41. Xue X, Chen Q, Zhao G, et al. The Overexpression of TGF- $\beta$ and $\mathrm{CCN} 2$ in Intrauterine Adhesions Involves the NF- $\kappa \mathrm{B}$
Signaling Pathway. PLoS One. 2015; 10(12): e0146159, doi: 10.1371/journal.pone.0146159, indexed in Pubmed: 26719893.

42. Su VYF, Lin CS, Hung SC, et al. Mesenchymal Stem Cell-Conditioned Medium Induces Neutrophil Apoptosis Associated with Inhibition of the NF- $\kappa$ B Pathway in Endotoxin-Induced Acute Lung Injury. Int J Mol Sci. 2019; 20(9), doi: 10.3390/ijms20092208, indexed in Pubmed: 31060326.

43. Kharaziha P, Hellström PM, Noorinayer B, et al. Improvement of liver function in liver cirrhosis patients after autologous mesenchymal stem cell injection: a phase I-II clinical trial. Eur J Gastroenterol Hepatol. 2009; 21(10): 1199-1205, doi: 10.1097/ MEG.0b013e32832a1f6c, indexed in Pubmed: 19455046.

44. Caplan H, Olson SD, Kumar A, et al. Mesenchymal Stromal Cell Therapeutic Delivery: Translational Challenges to Clinical Application. Front Immunol. 2019; 10: 1645, doi: 10.3389/ fimmu.2019.01645, indexed in Pubmed: 31417542.

Submitted: 10 May, 2020

Accepted after reviews: 21 September, 2020 Available as AoP: 30 September, 2020 